\title{
The Use of Fractal Dimension in Characterizing Tree Growth in Filled Epoxy Resin under Humid Condition
}

\author{
Rudi Kurnianto Member (Toyohashi University of Technology, rudi@boss.eee.tut.ac.jp) \\ Yoshinobu Murakami Member (Toyohashi University of Technology, murakami@eee.tut.ac.jp) \\ Naohiro Hozumi Member (Toyohashi University of Technology, hozumi@eee.tut.ac.jp) \\ Masayuki Nagao Member (Toyohashi University of Technology, nagao@tut.ac.jp)
}

Keywords : epoxy resin, filler, electrical tree, humidity, fractal dimension, damaged area

In this study, the influence of filler content, as well as humidity, on the fractal structure is discussed. Figure 1 shows the increasing tendency of fractal dimension as a function of tree length. When silica fillers had been introduced, the curve was shifted up both in humid and dry conditions. In another case, when humid condition had been introduced, the curve was shifted down both in specimens with and wihtout filler. It is suggested that the propagation characteristic of tree was consistent with its fractal dimension value. In our previous result; the tree propagation in epoxy-filler interface was accompanied by more branches than that in epoxy resin. Thus, fractal dimension has very close relationship with the number of branches. The introduction of filler brought the rise in fractal dimension due to the increase of branches.

In another case, the number of tree branches in humid condition was smaller than that in the dry condition. In dry conditions, the large number of tiny tree branches existing in the epoxy resin would bring the high fractal dimension in "without-filler" specimen. Also the large number of tree branches at the epoxy-filler interface would bring the higher fractal dimension in "with-filler" specimen. In humid conditions, the tree branches would be prevented, leading to the less fractal dimension compared to that in dry condition.

In case of humid conditions, the presence of moisture will reduce the dc resistivity and the dielectric strength, whereas it will slightly increase the permittivity of the composite. Instead of generating branch, the tree tends to propagate with less branches, leading to less fractal dimensions.

Figure 2 represents the relation between the tree length and fractal dimension with different filler contents. The higher filler contents would bring the larger number of tree branches at the

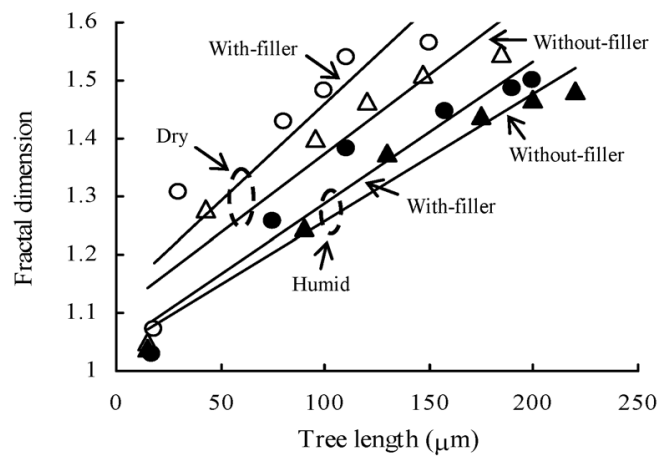

Fig. 1. Fractal dimension vs. tree length in epoxy resin under dry and humid conditions in without and with $8 \mathrm{phr}$ filler content

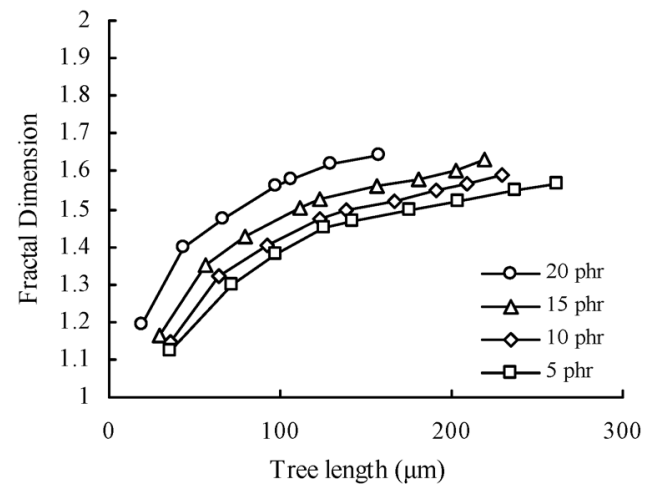

Fig. 2. Fractal dimension vs. tree length in epoxy resin with various content of fillers under ambient condition

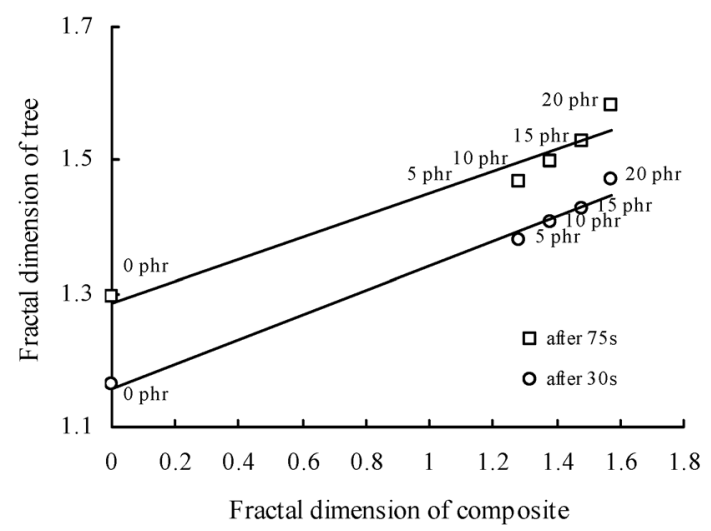

Fig. 3. Relation between fractal dimension of tree and composite under ambient condition $(30 \mathrm{~s}$ and $75 \mathrm{~s}$ after inception)

epoxy-filler interface, leading to a tree structure of higher fractal dimension. The higher filler content brought the faster change in tree shape from "treeing-like" to "branching-like" shape. The "fractal dimension of tree" can also be correlated to the "fractal dimension of composite material" as shown in Fig. 3. It is clear that the fractal dimension of the composite material increases as a function of filler content. As the fractal dimension of the tree also increases as a function of filler content, the good relationship between these two fractal dimension is quite reasonable. It is evident that the linear relationship stays unchanged with the tree propagated for $30 \mathrm{~s}$ and $75 \mathrm{~s}$, showing a very strong realationship between these two numbers. The higher filler content, with a large fractal dimension, would lead to more complex structure of the tree, bringing a large fractal dimension. 


\title{
The Use of Fractal Dimension in Characterizing Tree Growth in Filled Epoxy Resin under Humid Condition
}

\author{
Rudi Kurnianto* Member \\ Yoshinobu Murakami* Member \\ Naohiro Hozumi* Member \\ Masayuki Nagao* Member
}

\begin{abstract}
The structural response of tree growth in epoxy resin blended with silica filler has been investigated. The physical properties of the resin were varied by changing its filler content and exposing to humid air. The fractal dimension of the electrical tree and its relationship with filler content and humidity were determined. The damaged area of tree in various contents of filler was also estimated. It is considered that the filler would create such an obstruction to the tree growth both in humid and dry conditions. At the ambient condition, the more filler content, the more obstruction would be generated, leading to the significant suppression of tree growth. Likewise, the introduction of filler brought a rise in fractal dimension due to the increase of branches. It is concluded that the existence of filler makes the tree structure more complicated by introducing obstacles to tree propagation, leading to the high fractal dimension of the tree. In addition, it was found that the fractal dimension of the tree was very relational to the fractal dimension of the composite material including filler particles.
\end{abstract}

Keywords : epoxy resin, filler, electrical treeing, humidity, fractal dimension, damaged area

\section{Introduction}

Since electrical treeing is one of the main factors for ageing of electrical insulation, it has been studied for many years. Electrical treeing has complex structural characteristics that are difficult to describe using Euclidean geometry. As Mandelbrot ${ }^{(1)}$ established fractal geometry to describe the complexity of objects found in nature, trees can be appropriately described using fractal geometry and subsequently characterized by the fractal dimension. The fractal dimension can be determined, using box-counting $\operatorname{method}^{(2)}$, to be a non-integer value. Within this context, the structures of tree growth in filled epoxy resin have been studied extensively.

In the previous paper ${ }^{(3)}$, we clarified that humidity enhances the tree growth, and that the introduction of filler suppresses the growth. In these analyses, the tree growth was characterized only by the tree length. Here the tree length was defined as the distance between the tip of the needle electrode and the most distant tip of branch. In this case the two- (or three-) dimensional spread was ignored. On the other hand, in order to characterize the overall structure of tree patterns during their growth, many authors have been using fractal dimensions in addition to the above parameters. For example, Kudo et al. ${ }^{(4)}$ calculated the fractal dimension of electrical trees and found that the dimension increased as the tree grew. Champion et al. ${ }^{(5)}$ proposed a method for characterization of the spatial and temporal development of trees in the pin-plane electrode geometry. Naoe et al. ${ }^{(6)}$ performed fractal analysis of treeing process and correlated with the tree length and luminescence. Image analyses by pixel counting to characterize tree growth have also been carried out by Yoshimura et al. ${ }^{(7)}$, and

* Department of Electronic and Information Engineering., Toyohashi University of Technology

Hibarigaoka 1-1. Tempaku-cho, Toyohashi 441-8580
Cooper and Swingler ${ }^{(8)}$.

Such studies used homogeneous solid materials as specimens; however, materials are often mixed with a filler in order to upgrade thermal and mechanical properties. The existence of filler may affect the fractal parameters of the tree growing through the composite material. In this paper, the influence of filler content, as well as humidity, on the fractal structure is discussed.

\section{Experimental}

2.1 Epoxy Resin and Curing Process About $200 \mu \mathrm{m}$ thick leaf-like specimens were used to visualize tree growth along the resin-filler interface, as shown in Fig. 1. The epoxy resin employed was a blend of Araldite CY221 and HY2967 as the main resin and hardener, respectively. After blending the fillers with the epoxy resin and evacuating for 30 minutes, the compound was pasted onto the slide glass. The filler was a round-shaped silica with a diameter of about $50 \mu \mathrm{m}$. The needle electrode, made of a tungsten wire with $30 \mu \mathrm{m}$ in diameter, was placed with a gap of $1 \mathrm{~mm}$ from the ground electrode. The needle tip was formed by electrolytic polishing. Its tip radius and tip angle were $2-5 \mu \mathrm{m}$ and

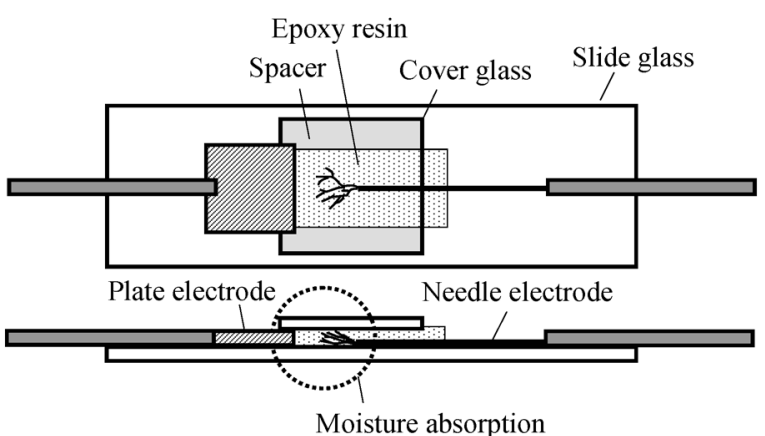

Fig. 1. Configuration of leaf-like specimen 
30 degree, respectively. The cover glass placed on the resin was kept at an appropriate distance from the surface of the slide glass using Ployimide film spacer with $200 \mu \mathrm{m}$ in thickness. The specimen was cured for 24 hours at room temperature. We prepared the specimens whose filler contents were fixed at $0,5,8$, 10,15 , and $20 \mathrm{phr}$ (parts per hundred resin) by weight ratio.

\subsection{Humidity Conditioning In order to accurately} control the humidity in a closed space, a chemical system which produces the desired equilibrium water vapor with a saturated salt solution $^{(9)}$ was employed. The saturated salt solution used was potassium sulfate $\left(\mathrm{K}_{2} \mathrm{SO}_{4}\right)$ to fix the relative humidity $(\mathrm{RH})$ at 97\%. We moisturized the specimens in this RH control cells for 240 hours prior to voltage application. For treeing test under dry condition, we dried the specimens in another control cell with $11 \%$ $\mathrm{RH}$ which is fixed by silica gel, also for about 240 hours prior to voltage application.

2.3 Observation of Electrical Tree Two steps of tree tests affected by environmental stress have been carried out using this composite material. In the first step we used specimens without and with $8 \mathrm{phr}$ of filler, both under humid and dry conditions. As a second step, specimens with several kinds of filler contents; 5, 10, 15, and $20 \mathrm{phr}$ were used. The test was carried out under amibient condition. The tree observation system for tree inception and propagation is shown in Fig. 2.

Tree inception voltage was defined as the voltage when the tree length observed by the CCD camera had exceed $10 \mu \mathrm{m}^{(10)}$. An ac ramp voltage $\left(60 \mathrm{~Hz}\right.$, increasing rate: $\left.0.2 \mathrm{kV}_{\mathrm{rms}} / \mathrm{s}\right)$ was applied to measure tree inception voltage for all conditions; humid $(97 \% \mathrm{RH})$, dry $(11 \% \mathrm{RH})$, and ambient. When applying the voltage, all of the specimens were placed in a vessel filled with silicone oil of 10 $\mathrm{mm}^{2} / \mathrm{s}(10 \mathrm{cSt})$ in viscosity under ambient temperature to prevent the surface flashover. As soon as a tree had been observed, the voltage was kept at the constant value to observe the tree propagation. In our experiment, the tree inception voltage was about $5 \mathrm{kV}_{\text {rms. }}$. We employed 7 specimens for each point of measurements.

2.4 Box-counting Method for Fractal Dimension The images of tree and filler were recorded by equipment, which was consisting of optical microscope, CCD (charge coupled device) camera, VTR (video tape recorder), and personal computer. The images were then enahanced by image processing. In comparison with homogeneous materials, the physical presence of filler such as in filled epoxy resin makes it difficult to observe the tree clearly when it developed through the filler-resin interfaces. Therefore in this study, we enhanced the tree image. The image before tree initiation was once recorded and substracted from the image after

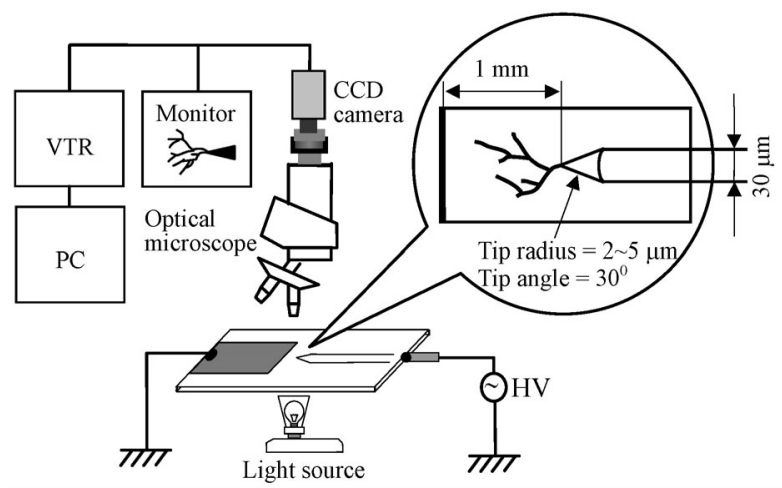

Fig. 2. Observation system of tree propagation tree propagation. As the former includes only the image of filler and the latter includes the images of both filler and tree, the image of the tree is discriminated after the subtraction process. The filler image is defined by the image of filled specimen which is not generated by treeing.

All of electrical tree propagations and filler images were structurally characterized by determining their fractal dimensions by box-counting method. It should be noted that the test object differs from background by color.

The procedure of fractal dimension determination on the test object images was done (made) by means of proper color separation (RGB channel, intensity, brightness, contrast), the masking procedure was made for color adjustment - black for the test object and white for background, as shown in Figs. 3-5. Fractal dimension can be determined by covering its image with boxes (squares) and subsequently evaluating how many squares are needed to cover fractal completely. The number of squares required to cover the fractal $N(r)$ is inversely proportional to the size of the squares $r$, as given by the next expression,

$$
d_{f}=(\log N(r) / \log (1 / r)
$$

where $N(r)$ is the number of repetitions of the reduced fractal pattern and $1 / r$ is the ratio of its reduction. Repeating this measurement with different sizes of squares will result into logarithmic function of square size (x-axis) and number of squares needed to cover fractal (y-axis), as shown in Fig. 6.
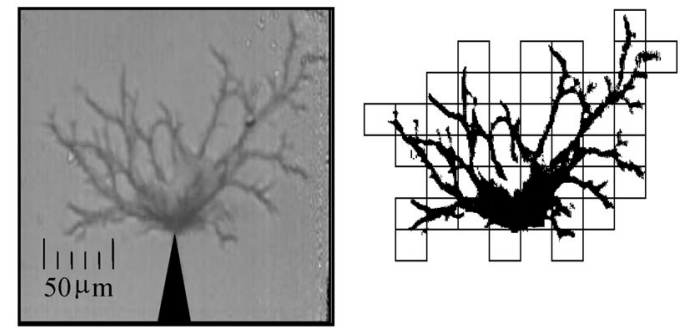

Fig. 3. A tree image of analyzed "without-filler" specimen under dry condition (left), the same image after masking (right)
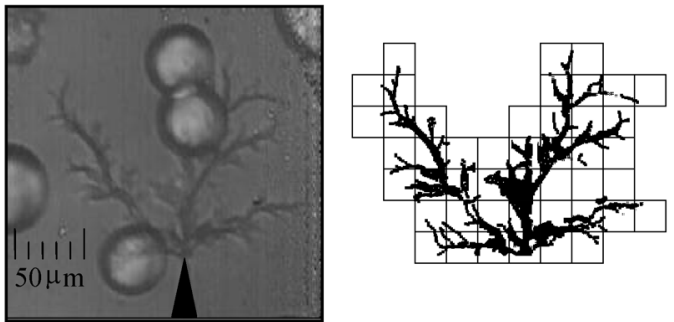

Fig. 4. A tree image of analyzed "with-filler" specimen under dry condition (left), the same image after masking (right)

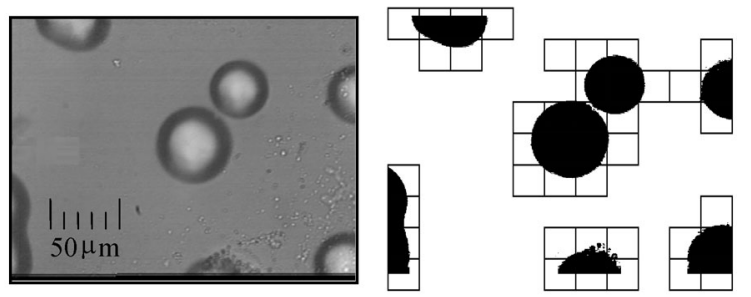

Fig. 5. An image of filled epoxy resin specimen blurred by microscope (left), the same image after masking (right) 


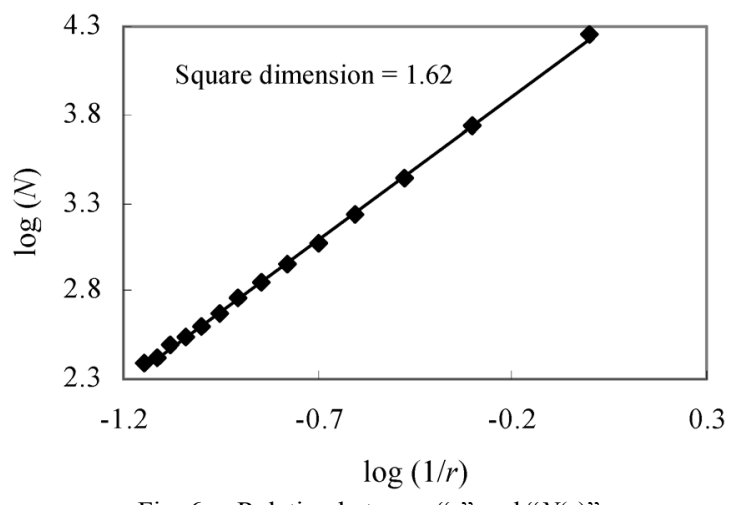

Fig. 6. Relation between " $r$ " and " $N(r)$ "

The slope of this function is reffered as square dimension ${ }^{(2)}$. Square dimension is taken as an appropriate approximation of fractal dimension. Moreover, we determined also the damaged area (or mass) caused by the tree by counting all pixels in the tree image. The damaged area was the minimum number of squares required for complete coverage. The resolution of our image processing gives the connection between image sizes and studied specimen size being approximately 1 pixel equivalent to an area of $156.86 \mu \mathrm{m}^{2}$.

\section{Results and Discussion}

Figure 7 shows the length of tree as a function of time under dry and humid conditions. The tree propagation of the specimen with filler was suppressed compared with the specimen without filler, both under dry and humid conditions. The mechanism for suppressing the tree propagation by filler can be explained in Fig. 8. As tree incepted as (a), and when it collided a filler as (b), it seemed that the tree extent was being suppressed. The inorganic filler would be more resistant against partial discharge compared with the resin. Therefore, it is considered that fillers would create such an obstruction to the tree propagation, and prevent tree from growing straight. Moreover, the difference between dielectric constant of the filler and epoxy resin would help branching accompanied by Maxwell stress, etc. ${ }^{(3)(10)(11)}$, leading to the imperfect adhesion at the interface. As pointed out in the previous paper $^{(12)}$, the quality of the adhesive bond between filler and resin determines the resistance to treeing incident. The higher resistance is presented by the filler which adhere strongly to the resin (shown by silane coupling agent treatment), while the imperfect adhesion (shown by release agent treatment) would lead to the tendency for treeing to occur. After propagating along the interface, the tree continuously propagates to another filler through the resin as (c)-(e), and finally leads to the breakdown (f).

A detail illustration of the tree colliding filler is also shown in Fig. 9. As the result in Ref. (13), the initial tree growth (a) would be expanded by the energy of electrons which flow in and out through the tip of the needle. Gas discharges would occur in the tree pipe when the tree reaches a certain length, and a certain diameter. According to the FDTG (field driven tree growth) model proposed by Champion et al. ${ }^{(5)}$, if the tree structure is conducting (at least during partial discharge activity), it will modify the local field condition, by effectively reducing the neelde-plate separation and increasing the apparent needle-plate radius. The growing tree can be approximated by a conducting hyperboloid of hyperbolic radius equal to tree length as represented by the equipotential lines. During tree growth, the hyperbolic-plate distance $d_{e}$, decreases as,

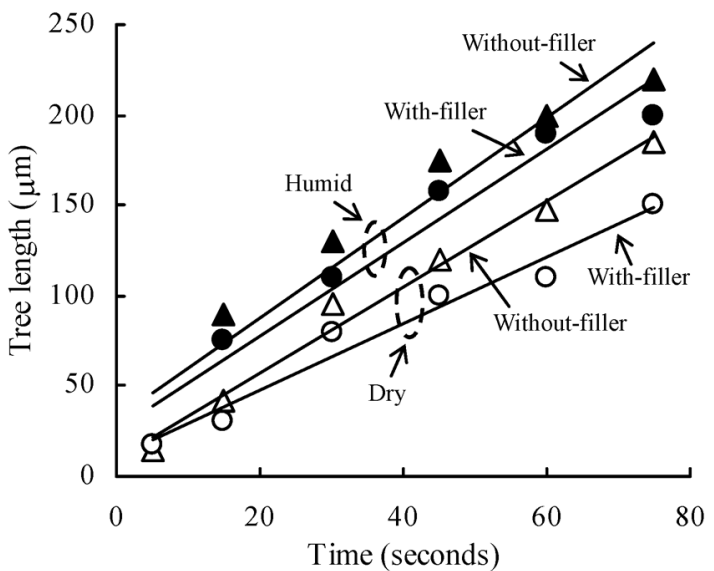

Fig. 7. Tree length vs. time in epoxy resin under dry and humid conditions in without and with $8 \mathrm{phr}$ of filler content

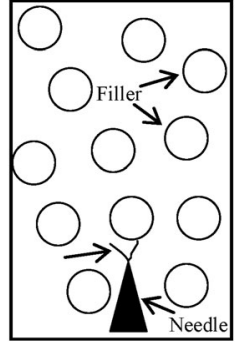

(a)

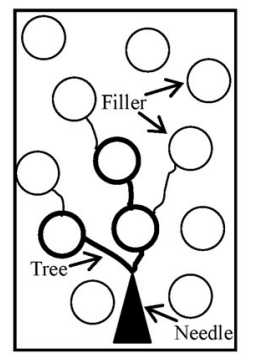

(d)

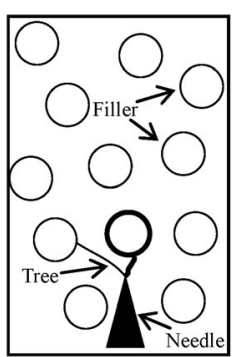

(b)

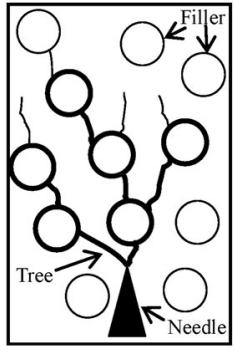

(e)

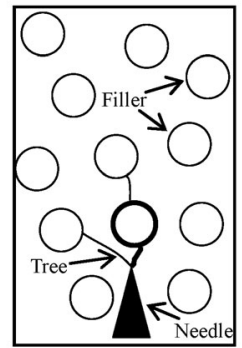

(c)

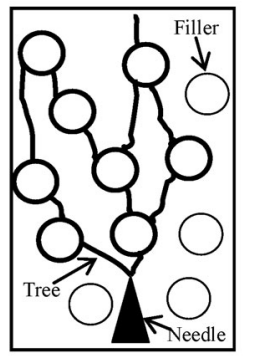

(f)
Fig. 8. The growth mechanism of tree in filled epoxy resin specimen

$d_{e}=d_{p}-r_{e}$. Here $d_{p}$ is the needle-plate distance and $r_{e}$ is the tree length. As another result in Ref. (13), when the top end of the tree meets filler (b), the filler would induce branching of the tree. The growth of the tree would slowed down once. At this moment, if the pressure in the tree pipe increased or the tree path acquired conductivity due to ions adhering to the tree wall, the gas discharge would not be maintained. When the pressure drops due to the dispersion of the gas in the pipe, or when the conductivity drops due to dispersion and extinction of ions on pipe wall gas discharge could occur again and tree would start to grow again. As the weak point at interface part, the tree would grow to the interface until completely cover the filler (c).

If we look back to Fig. 7, we can describe that; although the introduction of humid condition would bring moisture that promoted tree propagation, it seems that the filler still suppresses tree propagation.

The length of tree as a function of time with various contents of fillers under ambient condition is shown in Fig. 10. The higher filler content will generate the more obstruction to produce the more branching. On a global scale they may lead to significant 


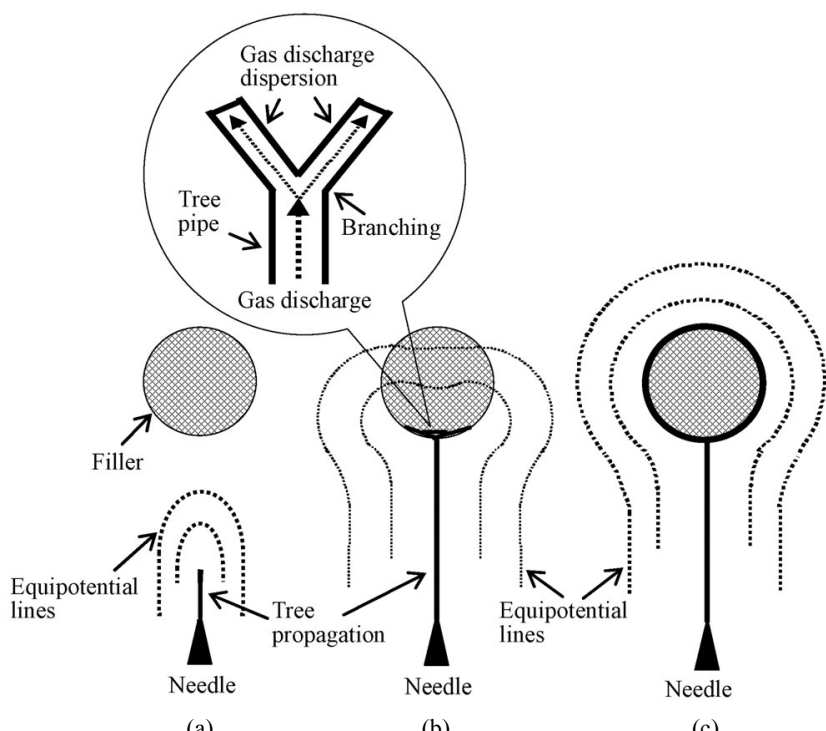

(a) (b)

(c)

Fig. 9. Branching mechanism by filler

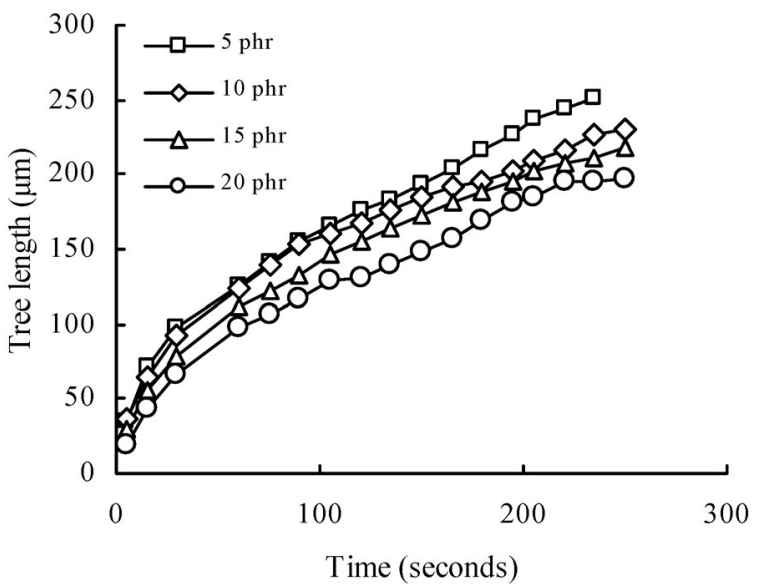

Fig. 10. The tree length vs. time in epoxy resin with various contents of fillers under ambient condition

suppression of tree propagation.

Figure 11 shows the increasing tendency of fractal dimension as a function of tree length. When silica fillers had been introduced, the curve was shifted up both in humid and dry conditions. In another case, when humid condition had been introduced, the curve was shifted down both in specimens with and without filler. It is suggested that the propagation characteristic of tree was consistent with its fractal dimension value. In our previous result ${ }^{(3)}$; the tree propagation in epoxy-filler interface was accompanied by more branches than that in epoxy resin as shown in Fig. 12. Thus, fractal dimension has very close relationship with the number of branches. The introduction of filler brought the rise in fractal dimension due to the increase of branches.

In another case, the number of tree branches in moisturized condition was smaller than that in non-moisturized condition as shown in Fig. 12, which coincides with the result shown in Fig. 11. In dry conditions, the large number of tiny tree branches existing in the epoxy resin would bring the high fractal dimension in "without-filler" specimen. Also the large number of tree branches at the epoxy-filler interface would bring the higher fractal dimension in "with-filler" specimen. In humid conditions, the tree branches would be prevented, leading to the less fractal dimension

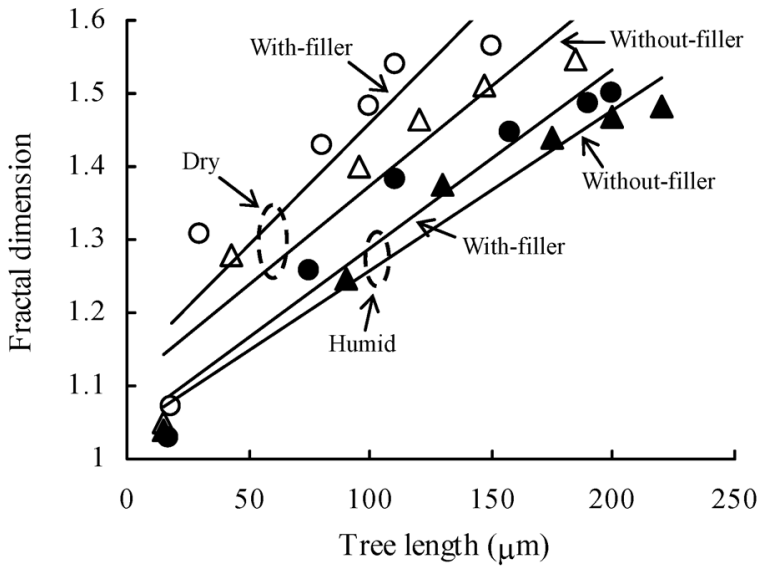

Fig. 11. Fractal dimension vs. tree length in epoxy resin under dry and humid conditions in without and with 8 phr filler content

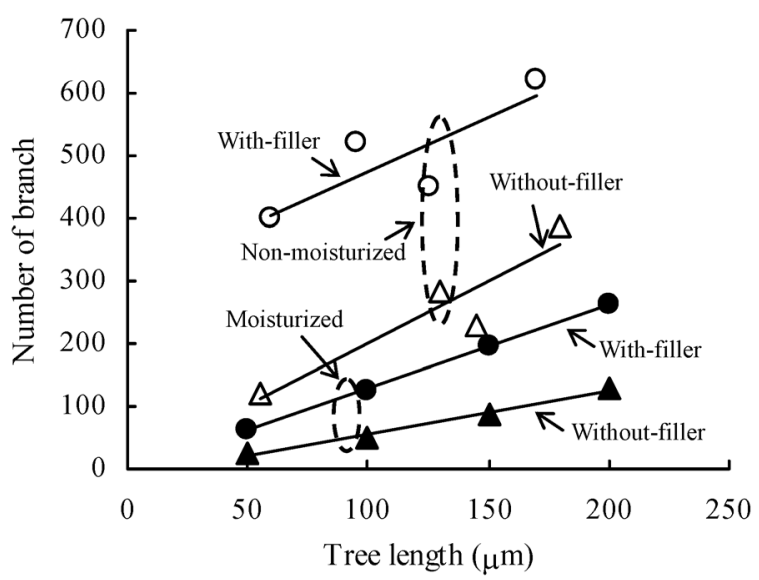

Fig. 12. Relation between tree length and number of tree branches in without and with 20 phr filler content

compared to that in dry condition.

The presence of moisture leads to the complex physical and chemical interactions at the interface. According to J.M. Seifert and H.C. Karner ${ }^{(11)}$ and N.H. Malik et al. ${ }^{(14)}$; the results of interactions at the large internal filler surfaces are an increase in the conductivity (or decrease of dc resistivity) and an intensification of the Maxwell-Wagner effect. These effects combined with the electrical stress can lead to the detachment of the filler-resin bond which results in a slightly increase of the permittivity and a decrease of dielectric strength of the composite. Therefore the tree tends to propagate with less branches, leading to the less fractal dimensions.

Figure 13 represents the relation between the tree length and fractal dimension with different filler contents. The higher filler contents would bring the larger number of tree branches at the epoxy-filler interface, leading to a tree structure of higher fractal dimension. In order to characterize the fractal dimension at the inception and propagation stages of tree growth, we divided the tree shape into two groups treeing-like and branching-like trees. According to our observation shown in Figs. 11 and 13, the fractal dimensions of tree patterns in two dimensional Euclidean space spreads approximately $1.01 \sim 1.24$ and $1.24 \sim 1.64$, respectively. At the inception, the tree grows nearly straight (linear), while at the stage of propagation, the tree grows with more branches. The higher filler content brought the faster change in tree shape from 


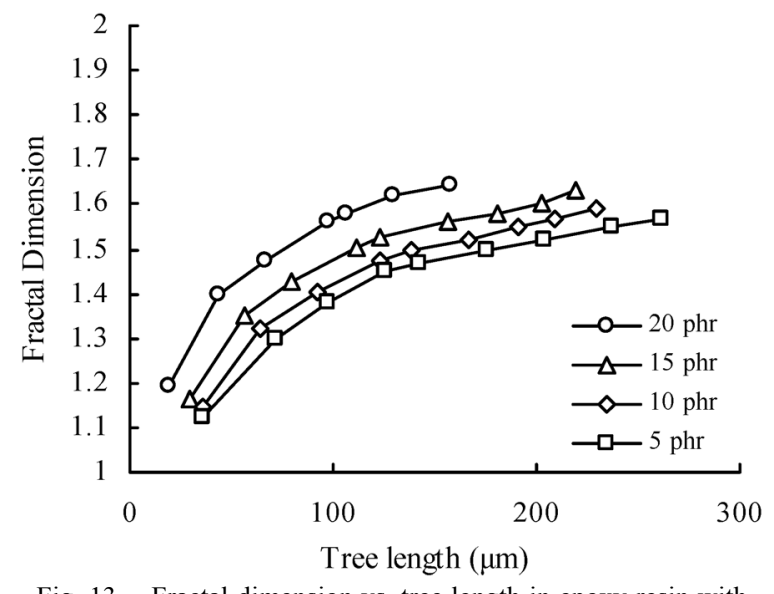

Fig. 13. Fractal dimension vs. tree length in epoxy resin with various content of fillers under ambient condition

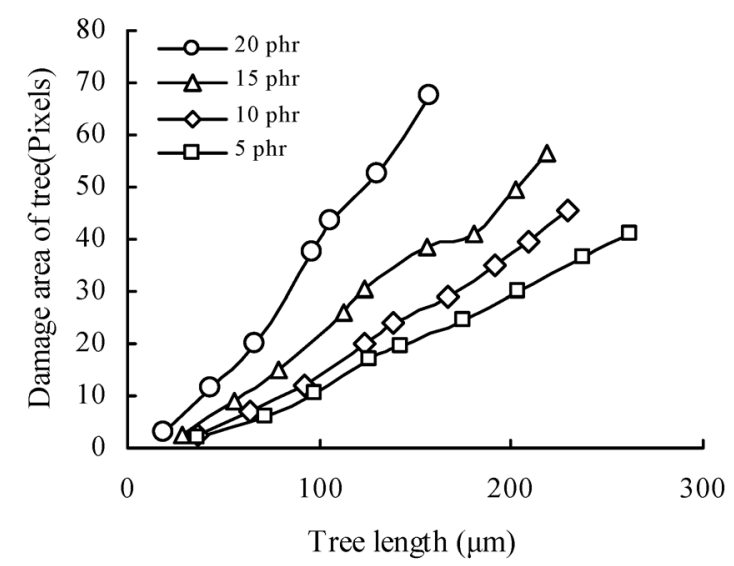

Fig. 14. Relationship between damage area and tree length, over various content of fillers under ambient condition

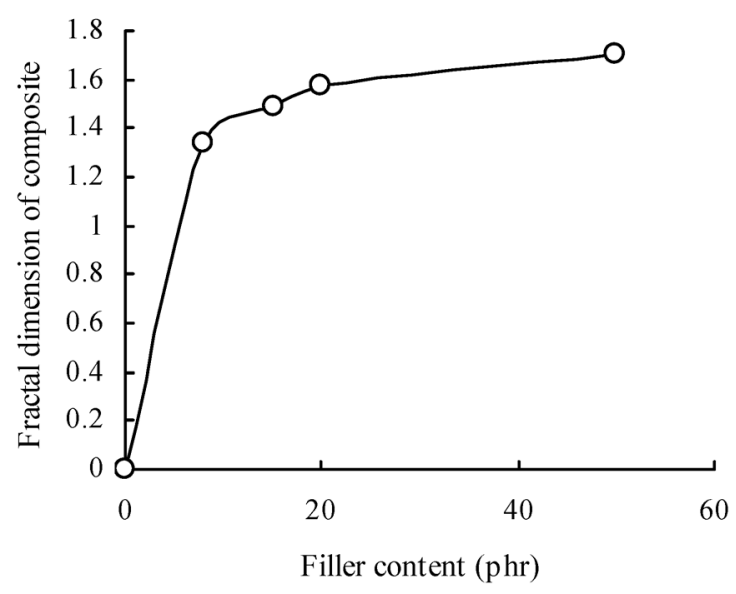

Fig. 15. Relation between filler content and fractal dimension of the composite under ambient condition

"treeing-like" to "branching-like" shape.

Figure 14 shows the relation between damaged area and tree length over various contents of fillers. The relationship indicates a significant change as the tree develops. A large amount of damaged area reflects the large density of tree branches. The highest proportion of the damaged area to tree length is seen with filler content of $20 \mathrm{phr}$.

Figure 15 shows the relation between filler content and fractal

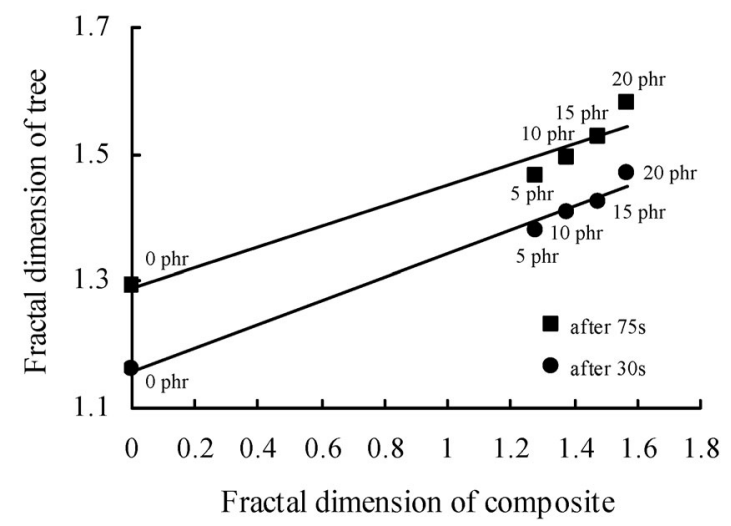

Fig. 16. Relation between fractal dimension of tree and composite under ambient condition (30 s and $75 \mathrm{~s}$ after inception)

dimension of the composite. As the diameter of filler is identical through various filler contents, it is natural that the diameter with $0 \mathrm{phr}$ of content is zero, and increases with content.

The "fractal dimension of tree" can also be correlated to the "fractal dimension of composite material" as shown in Fig. 16. It is clear that the fractal dimension of the composite material increases as a function of filler content. As the fractal dimension of the tree also increases as a function of filler content, the good relationship between these two fractal dimension is quite reasonable.

It is evident that the linear relationship stays unchanged with the tree propagated for $30 \mathrm{~s}$ and $75 \mathrm{~s}$, showing a very strong realationship between these two numbers. The higher filler content, with a large fractal dimension, would lead to more complex structure of the tree, bringing a large fractal dimension.

\section{Conclusions}

From our experiments, we concluded the following:

(1) A filler particle would create an obstruction to the tree propagation. Even though the introduction of humidity promoted tree propagation, it seems that the filler still suppresses tree propagation. The more filler content, the more obstruction will be generated, leading to the significant suppression of tree propagation.

(2) The introduction of filler raised the fractal dimension due to the increase in number of branches. The more filler content brought the higher fractal dimension.

(3) Fractal dimension in humid condition was lower than that in dry condition. This would have a relation with the reduction in dc resistivity and the dielectric strength when moisture was introduced to the specimen.

(4) Fractal dimensions of the composite material and tree showed a linear relationship, suggesting that the complexity of specimen structure is followed by the complexity of tree structure.

(Manuscript received Oct. 6, 2005, revised May 8, 2006)

\section{References}

(1) B. B. Mandelbrot : The Fractal Geometry of Nature, W. H Freeman, New York (1982)

(2) O. Zmeskal, M. Nezadal, and Buchnicek : "Fractal Analysis of Printed Structures", Conference of Polygrafia Academica 2000, CHTF STU Bratislava, pp.207-213 (2000-9) 
(3) M. Nagao, K. Oda, K. Nishioka, Y. Muramoto, and N. Hozumi : "Effect of Moisture on Treeing Phenomena in Epoxy Resin with Filler under ac Voltage”, Annual Rept. CEIDP, pp.951-954 (2002)

(4) K. Kudo and S. Maruyama : "Studies on the Fractal Properties of Electrical Trees", Proc. $3^{\text {rd }}$ Int. Conf. Properties and Application of Diel. Mater., pp.135-8 (1991)

(5) J. V. Champion, S. J. Dodd, and G. C. Stevens : "Analysis and Modelling of Electrical Tree Growth in Sysnthetic Resin over a wide Range of Stressing Voltage", J. Phys. D: Appl. Phys., Vol.27, pp.1020-30 (1994)

(6) M. Naoe, Y. Ehara, H. Kishida, and T. Ito : "The Fractal Analysis of the Treeing Process", IEEE Annual Report-Conference on Electrical Insulation and Dielectric Phenomena, San Fransisco, pp.779-782 (1996)

(7) N. Yoshimura, M. Yanagiwara, and L. G. Fan : "Diagnostics of Treeing Degradation by Image Processing", IEEE Trans. Electr. Insul., Vol.26, pp.314-7 (1991)

(8) J. M. Cooper and S. G. Swingler : "Image Analysis of Electrical Trees", $6^{\text {th }}$ Int. Conf. Dielectric Materials Measurements Applications, pp.9-12 (1992)

(9) L. Greenspan : "Humidity fixed points of binary saturated aqueous solutions", J. Rese. National Bureau of Standards-A. Physics \& Chemistry, Vol.81A, No.1 (1977)

(10) L. A. Dissado and J. C. Fothergill : Electrical Degradation and Breakdown in Polymers, G. C. Stevens, Ed., Peter Peregrinus London (1992)

(11) J. M. Seifert and H. C. Karner : "Dielectric Diagnostic of Moisture Induced Degradation Processes in Mineral Reinforced High-Voltage Composite Insulation", IEEE Annual Report- Conference on Electrical Insulation and Dielectric Phenomena, pp.825-828, San Fransisco (1996-10)

(12) M. Nagao, K. Oda, K. Nishioka, Y. Muramoto, and N. Hozumi : "Effect of Filler on Treeing Phenomenon in Epoxy Resin under ac Voltage", Proc. of 2001 ISEIM (01TH8544), Q-1, pp.611-614, Himeji, Japan (2001)

(13) N. Hozumi, T. Okamoto, and H. Fukugawa : "Simultanenous Measurement of Microscopic Image and Discharge Pulses at the Moment of Electrical Tree Initiation", Jpn J. Appl. Phys., Vol.27, No.4, pp.572-576 (1988-8)

(14) N. H. Malik, A. A. Al-Arainy, and M. I. Qureshi : Electrical Insulation in Power System, Marcel Dekker, Inc. (1998)

(15) N. Hozumi, T. Okamoto, and H. Fukugawa : "TEM Observation of Electrical Tree Paths and Microstructures in Polyethylene", Jpn. J. Appl. Phys., Vol.27, No.7, pp.1230-1233 (1988-7)

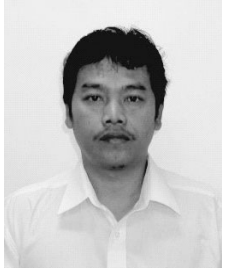

(Member) was born in Pontianak, Indonesia on May 27, 1967. He received his B.Eng. degree from Tanjungpura University Indonesia in 1994 and M.Eng. degrees both from Bandung Institute of Technology, Indonesia in 1998 and from Toyohashi University of Technology, Japan in 2005. He is now working towards his D.Eng degree in the Department of Electronic and Information Engineering at Toyohashi University of Technology. He has been working as a lecturer at Tanjungpura University since 1995. His research interests are in the areas of high voltage engineering. He is a member of IEE Japan and IEEE.

Yoshinobu Murakami (Member) was born in Japan on April 19, 1974. He was

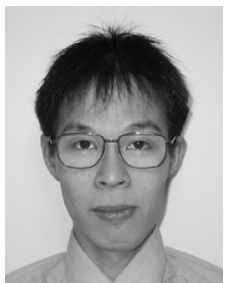
worked as a research assistant at the Department of Electrical Engineering, Nagano College of Technology from 2001 to 2003. He received a doctor engineering degree from Toyohashi University of Technology in late of March 2002. He is a research assistant at the Department of Electrical and Electronics Engineering, Toyohashi University of Technology Japan. He is a

member of IEE Japan.

Naohiro Hozumi

(Member) was born in Kyoto, Japan on April 2, 1957. He received his B.S., M.S. and $\mathrm{PhD}$ degrees in 1981, 1983 and 1990 from Waseda University. He was engaged in Central Research Institute of Electric Power Industry (CRIEPI) from 1983 to 1999 . He has been an associate professor at Toyohashi University of Technology since 1999. He has been engaged in the research in insulating materials and diagnosis for high voltage equipment. He was awarded in 1990 and 1999 from IEEJ for his outstanding research papers. He is a member of IEEE, IEE Japan, etc.

Masayuki Nagao

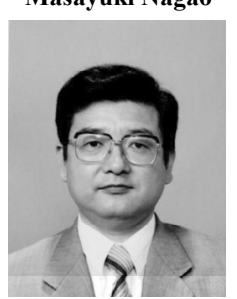

(Member) was born in Japan on April 16, 1950. He received the D.Eng. degree from Nagoya University in 1978. He became a Lecturer in 1980, an Associate Professor in 1985 and a Professor in 1998 in the Department of Electrical and Electronics Engineering of Toyohashi University of Technology. He is engaged primarily in research on high-field electrical properties of polymers and insulation degradation phenomena. $\mathrm{He}$ received a Paper Award from IEEJ in 1991. He is a member of the IEEJ, Japan Society of App. Phys., CIGRE, and Cryogenic Ass.of Japan. 